\title{
CEA (Carcinoembryonic Antigen) and CEACAM6 (CEA-Related Cell Adhesion Molecul 6) are Expressed in Psoriasis Vulgaris
}

\author{
Akihiko Fujisawa ${ }^{1}$, Kiyofumi Egawa ${ }^{2,3}$, Yumi Honda ${ }^{4}$, Masahide Kuroki ${ }^{5}$, Masatoshi Jinnin ${ }^{1}$ and \\ Hironobu Ihn ${ }^{*}, 1$ \\ ${ }^{I}$ Department of Dermatology and Plastic Surgery, Faculty of Life Sciences, Kumamoto University, Japan \\ ${ }^{2}$ Department of Dermatology, The Jikei University School of Medicine, Tokyo, Japan \\ ${ }^{3}$ Department of Microbiology, Kitasato University School of Allied Health Science, Sagamihara, Japan \\ ${ }^{4}$ Department of Surgical Pathology, Kumamoto University Hospital, Kumamoto, Japan \\ ${ }^{5}$ Department of Biochemistry, Faculty of Medicine, Fukuoka University, Fukuoka, Japan
}

\begin{abstract}
Carcinoembryonic antigen-related cell adhesion molecules (CEACAMs) belong to a group of mammalian immunoglobulin-related glycoproteins. The CEACAM family of proteins has been implicated in intracellular-signalingmediated effects that govern the growth and differentiation of normal and cancer cells.

In this study, the expression of CEACAMs was studied immunohistochemically in the skin of patients with psoriasis, using a panel of polyclonal (PoAb) and monoclonal (F34-187, F33-104, and F106-88) antibodies that recognize different epitopes of CEA and related molecules (CEACAMs), in comparison with the expression of cell differentiation and proliferation markers, such as involucrin, PCNA, Ki-67 and CK16. The expression of these molecules in adjacent parts without eruptions was also investigated for comparison.

The three CEACAMs, CEACAM1, CEA and CEACAM6, were expressed, limited to the upper part of the proliferated epidermal cells which expressed involucrin in the psoriatic lesions. Only the upper epidermal cell layers of the psoriatic lesions expressed these markers more highly than the adjacent normal skin. These results suggested that the expression of CEACAMs is related to epidermal cell de-differentiation in the diseased skin of psoriasis vulgaris.
\end{abstract}

Keywords: Psoriasis, CEA, CEACAM5, CEACAM6, CD66.

\section{INTRODUCTION}

The carcinoembryonic antigen (CEA) family consists of two subfamilies, the CEACAM subgroup and the pregnancy specific glycoprotein (PSG) subgroup [1, 2]. Recent studies have shown that CEACAMs are members of the immunoglobulin superfamily, and that they are composed of an $\mathrm{N}$-terminal domain $(\mathrm{N})$ and six very homologous immunoglobulin constant region-like domains (A1, B1, A2, B2, A3 and B3) [3, 4]. The CEACAMs in the human being consist of 18 genes and 11 pseudogenes on chromosome 19q13.2.

CEACAMs are now recognized as being important not only as tumor markers but also as cell adhesion molecules $[5,6]$. Furthermore CEACAMs are considered to be capable of transmitting the signals that result in a variety of effects depending on the tissue, including tumor suppression, tumor promotion, angiogenesis, neutrophil activation, lymphocyte activation and the cell cycle regulation [1].

CEACAMs expression has been reported not only in normal and malignant mucosa of the gastrointestinal tract,

*Address correspondence to this author at the 1-1-1 Honjo, Kumamoto 8608556, Kumamoto, Japan; Tel: +81-96-373-5233; Fax: +81-96-373-5235;

E-mail: ihn-der@kumamoto-u.ac.jp but also in some inflammatory diseases [3] and epithelial neoplasms [7] of the skin, gall bladder and extrahepatic biliary tract [8]. CEACAMs are also expressed in follicular keratinocytes [3,9] and sweat gland apparatus $[10,11]$ in normal skin.

Concerning the psoriatic skin, CEACAM1 has been reported to be expressed in outer epidermal cell layers of the psoriatic skin [12]. Over expression of CEA was also demonstrated immunohistochemically [3, 13]. However, it remains unclear whether other CEACAMs are expressed in psoriatic skin.

In the present study, we aimed to evaluate the expression of CEACAMs by immunoblotting as well as immunohistochemical staining analysis. In the present study, we also developed an antigen retrieval method suitable for in situ CEACAMs expression in formalin-fixed materials.

\section{MATERIAL AND METHODS}

\section{Materials}

Totally 37 tissue samples of psoriasis vulgaris (28 formalin-fixed and 9 frozen specimens) were obtained from 28 patients who received biopsy in the Department of Dermatology and Plastic Surgery at Kumamoto University 
Hospital. Written informed consent to participate in the study was obtained from all the patients, which was approved by the institutional review board.

For histological and immunohistochemical analyses, 28 specimens were fixed in $10 \%$ formalin and embedded in paraffin. Nine of 28 specimens were divided into two pieces, one of which was frozen in liquid nitrogen and kept at $-80^{\circ} \mathrm{C}$ for further analysis.

\section{Antibodies Used}

Three mouse monoclonal antibodies (F34-187, F33-104 and F106-88; developed by Kuroki et al.) and one rabbit polyclonal antibody raised against human CEACAMs (DakoPoAb) were used as primary antibodies. Epitope mapping analysis, using CEA and related antigens and various CEA and related recombinant proteins, has revealed the specificity of the monoclonal antibodies used here $[14,15]$.

F34-187, which recognizes an epitope on domain $\mathrm{N}$ of CEA, reacts with CEA, CEACAM1 and CEACAM6 [14, 15]. F33-104, which recognizes an epitope on the B3 domain of CEA, seems to be specific for CEA $[14,15]$. F106-88 reacts only with CEACAM6 $[14,15]$. Dako-PoAb reacts with CEA and other CEACAM members.

A well-characterized PoAb against involucrin (Paesel), MoAbs against PCNA (Dako), Ki-67 (MIB-1, SANTA CRUZ BIOTECHNOLOGY, INC.) andCK16 (Novocastra) were also used as proliferation and/or differentiation markers to investigate the histological localization of the CEACAMs in comparison with the molecules.

\section{Immunoblotting Analysis}

Four frozen samples of psoriasis vulgaris and 3 of normal skin were used for immunoblotting analysis. The samples were frozen in liquid nitrogen and kept at $-80^{\circ} \mathrm{C}$. One frozen sample of rectal carcinoma was also used for positive control and human fibroblasts cultured in modified Eagle's medium supplemented with $10 \%$ fetal calf serum (FCS) and $50 \mu \mathrm{g} / \mathrm{ml}$ amphotericin for negative control.

The specimens were homogenized and added phosphatidylinositol-specific phospholipase C (PI-PLC; Sigma) to cleave off the GPI-anchored proteins, such as CEA and CEACAM6, from the extracellular membrane. Then $20 \mu 1$ of the supernatant of the sample were exposed to $7.5 \%$ SDSpolyacrylamide gel electrophoresis before transferring to a nitrocellulose membrane. Blocking was achieved by incubating the membrane in $3 \%$ skimmed milk/Tris buffered saline with $0.2 \%$ Twin-20 (TBST) overnight at $4{ }^{\circ} \mathrm{C}$.

Dako-PoAb was applied at the dilution of 1:1000 and incubated overnight at $4{ }^{\circ} \mathrm{C}$, after which the membrane was incubated with goat anti-rabbit IgG-HRP for $60 \mathrm{~min}$. Likewise, F33-104 (specific for CEA) and F106-88 (specific for CEACAM6) were applied at the dilution of 1:1000 and incubated overnight at $4{ }^{\circ} \mathrm{C}$, after which the membranes were incubated with goat anti-mouse IgG-HRP for $60 \mathrm{~min}$. Membranes were washed with TBST and signals detected using the enhanced chemiluminescence system (Amersham Biosciences). $\beta$-actin was used as a loading control.

\section{Immunohistochemical Staining Analysis}

Immunohistochemical staining for the expression of CEACAMs and the proliferation/differentiation markers was performed with Vectastain ABC kit (Vector Laboratories) on serial sections of frozen and/or formalin-fixed specimens.

In regard to the frozen material, $4 \mu \mathrm{m}$ cryostat sections were air-dried and fixed in acetone at $4{ }^{\circ} \mathrm{C}$ for $10 \mathrm{~min}$, whereas in regard to formalin-fixed material, after the sections were deparaffinized and rehydrated, antigen retrieval of CEACAMs was achieved by microwave treatment for $10 \mathrm{~min}$ in EDTA (1 mmol/1, pH6.0), followed by cooling at room temperature for $60 \mathrm{~min}$. After being washed in phosphate-buffered saline (PBS), the sections were treated with $3 \%$ hydrogen peroxide solution to reduce endogenous peroxidase activity. Those slides were incubated with the respective primary antibodies at $4^{\circ} \mathrm{C}$ overnight, washed in phosphate-buffered saline, treated with biotinylated anti-mouse (for MoAbs) or anti-rabbit (for Dako-PoAb) IgG (Vector Laboratories) for $30 \mathrm{~min}$ at room temperature, and rewashed. After $60 \mathrm{~min}$ of incubation with the avidin-biotin peroxidase complex (ABC, Vector Laboratories), the sections were washed in phosphate-buffered saline, developed in $0.05 \%$ diaminobenzidine diluted in phosphate-buffered saline with $0.01 \%$ hydrogen peroxide, dehydrated, and mounted.

11C2A4 (hybridoma protein; for F34-187, F33-104 and F106-88), normal mouse (Cappel Division, Organon Teknika Co.; for MoAbs), or rabbit serum (Cappel Division, Organon Teknika Co.; for PoAbs) were used as the negative controls. For positive controls, we examined normal eccrine glands and ducts in each section.

\section{Double Immunoenzymatic Staining Analysis}

Since the expression of CEACAMs was restricted to the upper epidermal cell layers, we performed the double immunoenzymatic staining analysis to compare the expression of the CEACAMs with that of involucrin, a well-characterized differentiation marker for the epidermal keratinocytes.

In brief, the sequential immunostaining procedure was carried out by first performing immunoperoxidase staining against F34-187, the monoclonal antibody attaining the clearest staining in our protocol, using the ABC method with a Vectastain ABC Kit (Vector Laboratories), and then the immunoalkaline phosphatase-antialkaline phosphatase (APAAP) method against involucrin (PoAb) was conducted with an APAAP Kit (Zymed Laboratories.). The sections were stained for peroxidase with diaminobenzidine and then stained with second primary antibody. As substrate solution for the alkaline phosphatase reaction, naphthol-AS-phosphatase (Sigma) and Fast Blue BB (Sigma) were dissolved in dimethylformamide and diluted with $0.1 \mathrm{~mol} / 1$ Tris buffer, $\mathrm{pH}$ 8.2. To inhibit endogenous alkaline phosphatase activity, the incubation medium was supplemented with $0.25 \mathrm{mmol} / \mathrm{l}$ levamisole (Sigma).

\section{RESULTS}

\section{Immunoblotting Analysis}

Immunoblotting analysis revealed that the expression of both CEA and CEACAM6was up-regulated in psoriatic skin compared to normal skin, in which CEACAM6 expression was detected, but not CEA (Fig. 1). 
Fibroblast Psoriasis Normal skin Rectal carcinoma

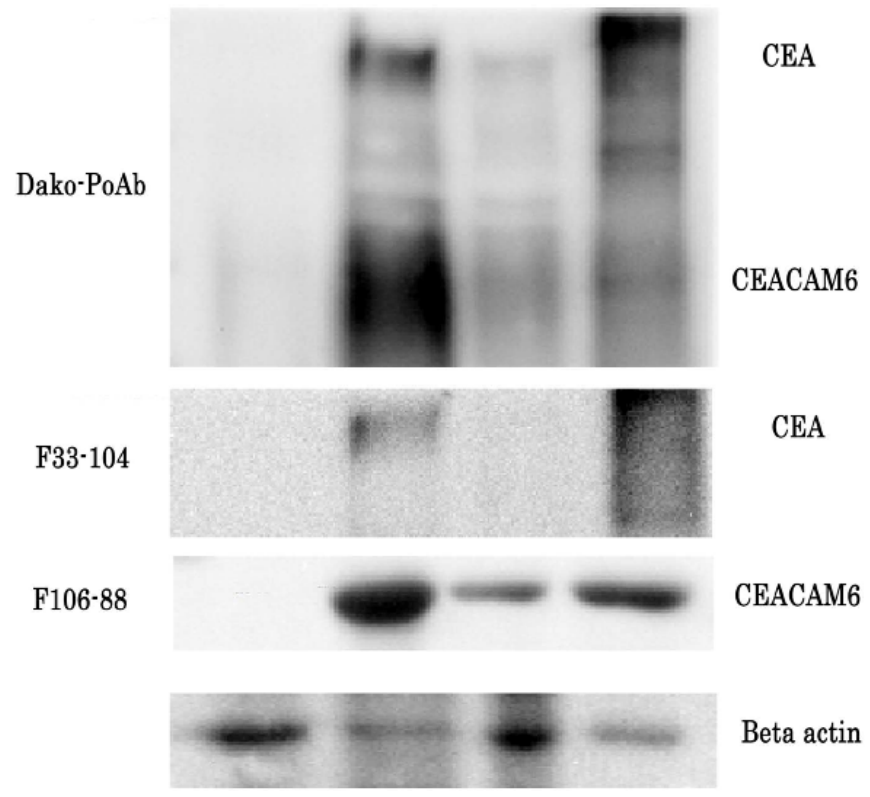

Fig. (1). Immunoblotting analysis of CEA and CEACAM6 expression in dermal fibroblasts, psoriasis skin, normal skin and rectal carcinoma. With F33-106 (specific for CEA), positive signal was seen only in psoriatic skin, whereas positive signal was seen in both psoriasis and normal skin with F106-88 (specific for CEACAM6), with much stronger signal in psoriatic skin than in normal skin. The $\beta$-actin was used as a loading control.

\section{Immunohistochemical Staining Analysis}

Using a Dako-PoAb against CEACAMs, the histological distribution of all the CEACAM-expressing cells was visible in the psoriatic skin. The expression was obtained in the epidermal keratinocytes only in the upper epidermal cell layers, while no expression was seen in the basal and lower prickle cell layers and in perilesional normal skin (Fig. 2). Positive signal was also seen on neutrophils in Munro's microabscess and some dermal and epidermal inflammatory infiltrating cells; the infiltration of the CEACAM-expressing inflammatory cells was restricted to the dermis just beneath the epidermis expressing the CEACAM, and was the denser infiltration of the CEACAM-expressing inflammatory cells seen in the dermis where the stronger expression of the CEACAM was seen in the overlying epidermis (Fig. 2). Eccrine sweat glands and ducts were also positive for the antibody.

Heamatoxylin and eosin stain shows hyperkeratosis, parakeratosis, acanthosis and Munro's microabcess (Fig 3a). In higher magnification, both membranous and cytoplasmic expression of the CEACAMs was seen in the epidermal keratinocytes (Fig. 3b). Similar expression pattern was also seen with the F34-187 and F33-104 (Fig. 3c) in the epidermal keratinocytes, suggesting that CEA was expressed in the psoriatic keratinocytes and this was confirmed by the result of immunoblotting analysis. Neither neutrophils in Munro's microabscess nor inflammatory infiltrating cells showed positive signals with the F33-104, suggesting that CEA was not expressed in these cells (Fig. 3c).

Using F106-88 (specific for CEACAM6), negative (Fig. 3d) to weak (Fig. 4) signal was obtained in the epidermal keratinocytes, depending on the samples studied, in contrast to that strong expression was seen by immunoblotting analysis using the same antibody as mentioned above. Neutophils in Munro's microabscess and inflammatory infiltrating cells were positive for F106-88, suggesting that CEACAM6 was expressed in the cells.



Fig. (2). Immunohistochemistry with Dako-PoAb shows strong CEACAM expression in the epidermal keratinocytes restricted to the upper epidermal cell layers, inflammatory infiltrating cells, and eccrine glands (x 20).

Up-regulated expression of PCNA, Ki-67, CK16 and involucrin was also shown in the epidermal keratinocytes of the psoriatic skin: PCNA and Ki-67 were expressed in the keratinocytes of basal and lower prickle cell layers, while CK16 and involucrin were expressed in middle to upper epidermal cell layers (Fig. 5).

The expression of the CEACAM was restricted to more de-differentiated. This finding was well demonstrated by double immunoenzymatic staining analysis (Fig. 6).

\section{DISCUSSION}

Using a panel of antibodies that recognize different epitopes of CEACAMs, we clearly demonstrated that CEA and CEACAM6 were overexpressed in inflamed psoriasis skin. The expression was detected in membrane and cytoplasm of the epidermal keratinocytes whose distribution was restricted to the upper cell layers of the acanthotic epidermis.

While, even though our immunoblotting analysis showed strong expression of CEACAM6 with F106-88 (MoAb specific for CEACAM6), our immunohistochemical analysis showed only a week positive signal (some lesions were even negative) with the same antibody in the epidermal keratinocytes. The reason for this weak staining could be because F106-88 antibody is not suitable for detecting naive protein, in contrast to its high immunoreactivity in immunoblotting.

Infiltrating inflammatory cells showed strong positive signal for not only Dako-PoAb and F34-187 but also F10688 , suggesting that CEACAM6 was strongly expressed in the inflammatory cells.

Immunohistochemical and double immunoenzymatic revealed that the CEACAMs expression correlated with the overexpression of involucrin, PCNA, Ki-67 and CK16. 

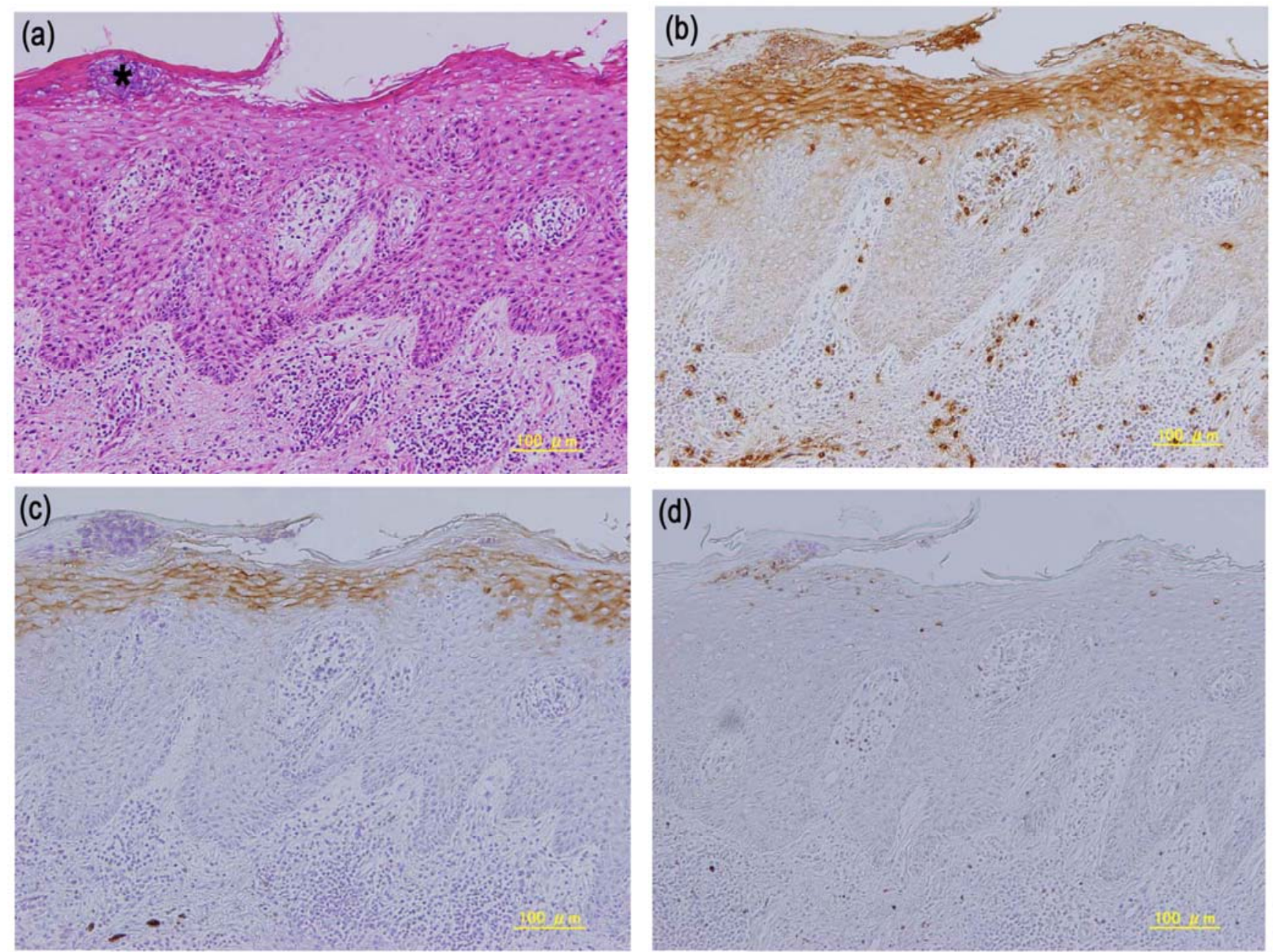

Fig. (3). Representative staining for CEACAMs in psoriasis skin. Haematoxylin and eosin stain (a) shows hyperkeratosis, parakeratosis, acanthosis and Munro's microabscess (*). Lymphocytes and a few neutrophils are present in the perivascular infiltrates. In immunohistochemical analysis, Dako-PoAb (b) shows positive signal in keratinocytes restricted to the upper epidermal cell layers and inflammatory infiltrating cells, including those in Munro's microabscess (*); F33-104 (c) shows positive signal in the epidermal keratinocytes and eccrine ducts, but not in the inflammatory infiltrating cells;F106-88 (d) shows positive signal only a few inflammatory infiltrating cells. Bar 100 $\mu$ m.

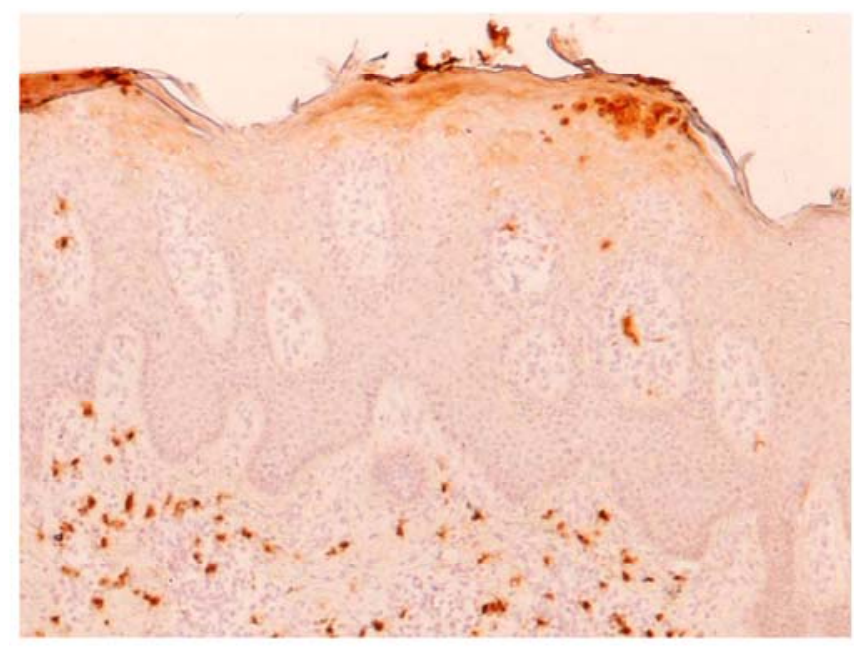

Fig. (4). Immunohistochamical staining with F106-88 showed week to moderate positive signal in keratinocytes of upper epidermal cell layers, in addition to the strong signal in inflammatory infiltrating cells (x 40).
These results suggest that both CEA and CEACAM6 are induced on epidermal keratinocytes in psoriasis lesions, and that the expression may be associated with the state of dedifferentiation of the actively proliferating keratinocytes of the disease. Up-regulated expression of CEA and other CEACAM members only in the upper epidermal cell layers was also demonstrated in keratinocytic neoplasma [7] and verruca vulgarisin in our previous studies [7,16], suggesting the differentiation-related expression of these molecules also exists in the proliferatively active keratinocytes in the neoplastic skin conditions.

It is currently well known that CEA and CEACAM6 are up-regulated in a wide variety of human cancers, including colon, breast, pancreatic, and lung cancer [17]. Functional analyses have indicated that CEA and CEACAM6 can inhibit differentiation [18] and anoikis [19] of a number of different cell lines. An inverse correlation between the cell surface levels of CEA and CEACAM6 and the degree of differentiation of the tumors has also been shown [20].

CEACAM1 was reported to be expressed only in outer epidermal cell layers in association with Munro's 

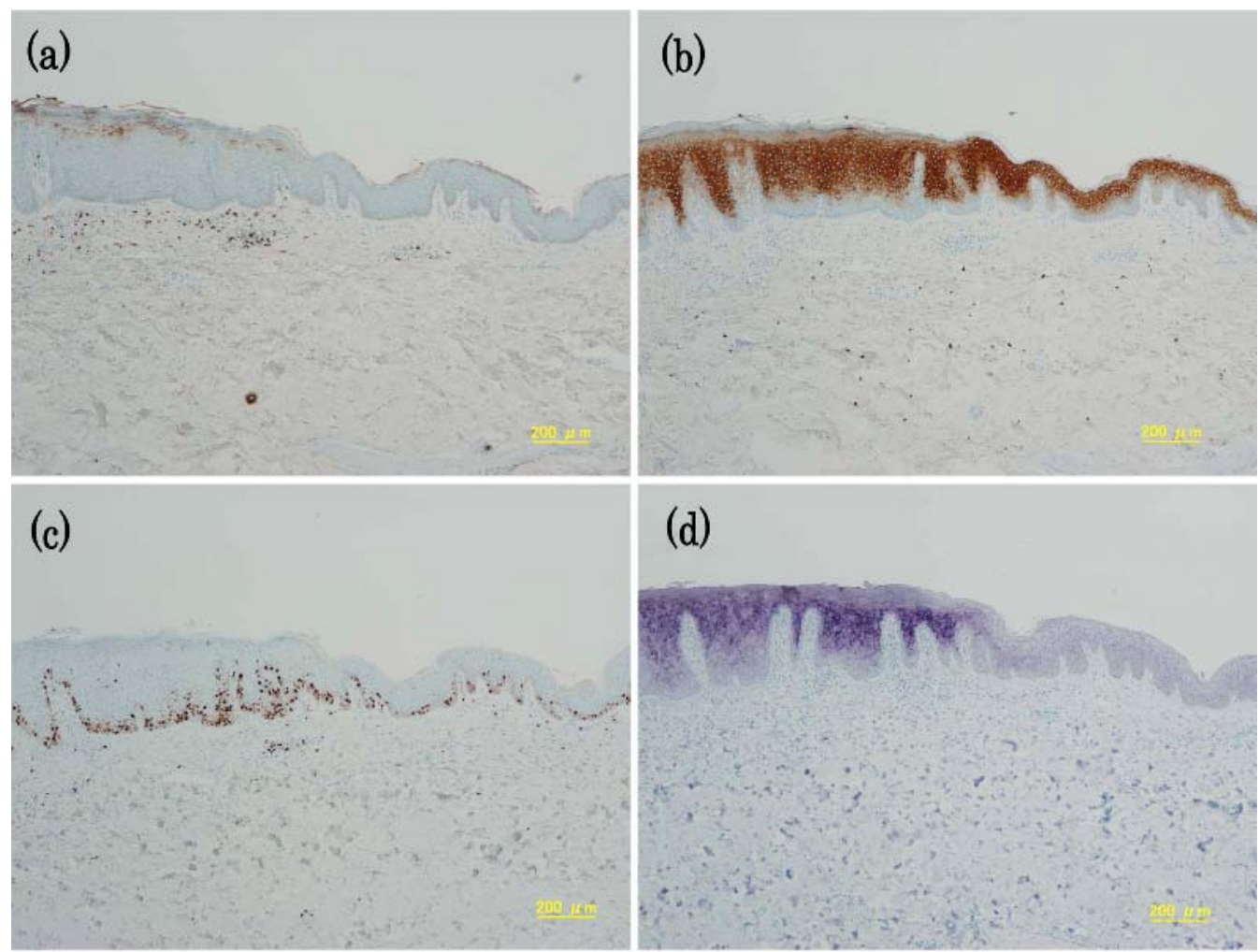

(d)

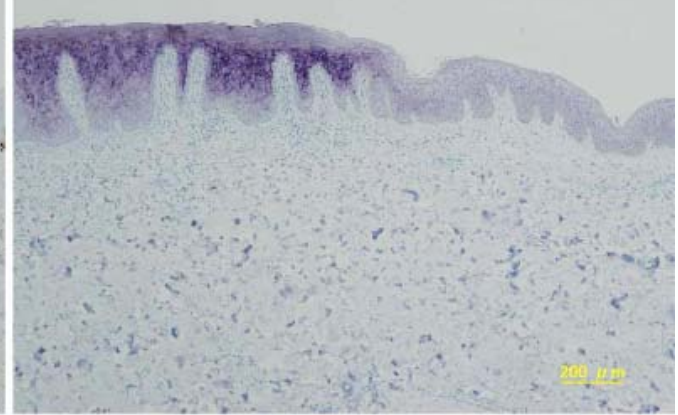

Fig. (5). Immunohistochemical staining for CEACAMs (a), involucrin (b), Ki-67 (c) and CK16 (d). (a) Membranous and cytoplasmic expression of CEACAMs is seen on keratinocytes only in the upper part of the epidermis. (b) Expression of involucrin existed at the lower epidermal cell layers. (c) In psoriasis lesions, overexpression of Ki-67 is seen in the nuclei in the basal and lower epidermal cell layers. (d) Expression of CK16 was seen only in the psoriasis lesions. Bar $200 \mu \mathrm{m}$.

microabscess [12]. However, in the present study, CEA was expressed diffusely in the upper epidermal cell layers, not only in association with the Munro's microabscess.



Fig. (6). Double immunoenzymatic staining with F34-187 (avidinbiotin peroxidasecomplex) and involucrin (alkaline phosphataseantialkaline phosphatase) shows the CEACAM expression (brown) exists at the epidermal cell layers above that of involucrin (blue) in psoriasis lesion. Neutrophils in Munro's microabcess also express the CEACAM (brown). Bar $100 \mu \mathrm{m}$.

Indeed these limited data are insufficient to elucidate true roles of CEACAMs in the pathogenesis of psoriasis vulgaris.
Interstingly, immunohistological studies have failed to show CEACAMs expression in atopic dermatitis [12], suggesting the difference of underlying pathogenesis of these skin disorders. In fact, it is generally accepted that the immune response in atopic dermatitis is mediated by Th2 lymphocytes, whereas Th1 cells are important in the pathogenesis of psoriasis [21]; it has recently been revealed that both CEA and CEACAM6 are major target for Smad3mediated TGF- $\beta$ signaling [22]; and that IFN- $\gamma$ or oncostatin $M$ induces the expression of CEACAM1 [12].
ABBREVIATIONS
CEA $=$ Carcinoembryonic antigen
CEACAM $=$ CEA-related cell adhesion molecule
$\mathrm{MoAb}=$ Monoclonal antibody
$\mathrm{PoAb}=$ Polyclonal antibody

\section{CONFLICT OF INTEREST}

The authors confirm that this article content has no conflict of interest.

\section{ACKNOWLEDGEMENTS}

Declared none.

\section{REFERENCES}

[1] Skubitz K, Skubitz A. Interdependency of CEACAM-1, -3, -6, and -8 induced human neutrophil adhesion to endothelial cells. J Transl Med 2008; 6: 78 
[2] Beauchemin N, Draber P, Dveksler G, et al. Redefined nomenclature for members of the carcinoembryonic antigen family. Exp Cell Res 1999; 252: 243-9.

[3] Egawa K, Honda Y, Kuroki M, Inaba Y, Ono T. Carcinoembryonic antigen and related antigens expressed on keratinocytes in inflammatory dermatoses. Br J Dermatol 1996; 134: 451-9.

[4] Gray-Owen S, Blumberg R. CEACAM1: contact-dependent control of immunity. Nat Rev Immunol 2006; 6: 433-46.

[5] Gold P, Freedman S. Demonstration oftumor-specific antigens in human colonic carcinoma by immunological tolerance and absorption techniques. J Exp Med 1965; 121: 439-62.

[6] Benchimol S, Fuks A, Jothy S, Beauchemin N, Shirota K, Stanners C. Carcinoembryonic antigen, a human tumor marker, functions as an intercellular adhesion molecule. Cell 1989; 57: 327-34.

[7] Egawa K, Honda Y, Ono T, Kuroki M. Immunohistochemical demonstration of carcinoembryonic antigen and related antigens in various cutaneous keratinous neoplasms and verruca vulgaris. $\mathrm{Br} \mathbf{J}$ Dermatol 1998; 139: 178-85.

[8] Maxwell P, Davis R, Sloan J. Carcinoembryonic antigen (CEA) in benign and malignant epithelium of the gall bladder, extrahepatic bile ducts, and ampulla of Vater. J Pathol 1993; 170: 73-6.

[9] Honda Y, Egawa K, Kuroki M, Ono T. Hair cycle-dependent expression of a nonspecific cross reacting antigen (NCA)-50/90like molecule on follicular keratinocytes. Arch Dermatol Res 1997; 289: 457-65.

[10] Metze D, Grunert F, Neumaier M, et al. Neoplasms with sweat gland differentiation express various glycoproteins of the carcinoembryonic antigen (CEA) family. J Cutan Pathol. 1996; 23: $1-11$.

[11] Metze D, Soyer H, Zelger B, et al. Expression of a glycoprotein of the carcinoembryonic antigen family in normal and neoplastic sebaceous glands. Limited role of carcinoembryonic antigen as a sweat gland marker. J Am Acad Dermatol 1996; 34: 735-44.

[12] Rahmoun $\mathrm{M}$, Molès $\mathrm{J}$, Pedretti $\mathrm{N}$, et al. Cytokine-induced CEACAM1 expression on keratinocytes is characteristic for psoriatic skin and contributes to a prolonged lifespan of neutrophils. J Invest Dermatol 2009; 129: 671-81.
[13] Hagemeier H, Bhardwaj R, Grunert F, et al. Carcinoembryonic antigen and related glycoproteins in psoriasis. Pathobiology 1993; 61: 19-24

[14] Kuroki M, Arakawa F, Higuchi H, Haruno M, Wakisaka M, Matsuoka Y. Immunological and biochemical characterization of the nonspecific cross-reacting antigen epitopes using twenty-three monoclonal antibodies. Hybridoma 1991; 10: 557-74.

[15] Kuroki M, Arakawa F, Haruno M, et al. Biochemical characterization of 25 distinct carcinoembryonic antigen (CEA) epitopes recognized by 57 monoclonal antibodies and categorized into seven groups in terms of domain structure of the CEA molecule. Hybridoma 1992; 11: 391-407.

[16] Egawa K, Honda $\mathrm{Y}$, Ono $\mathrm{T}$, Kitasato $\mathrm{H}$, Kuroki M. The glycoprotein of the carcinoembryonic antigen (CEA) gene family expressed on epithelial keratinocytes in viral warts. Arch Dermatol Res 1998; 290: 453-7.

[17] Ordonez C, Zhai A, Camacho-Leal P, Demarte L, Fan M, Stanners C. GPI-anchored CEA family glycoproteins CEA and CEACAM6 mediate their biological effects through enhanced integrin $\alpha 5 \beta 1$ fibronectin interaction. J Cell Physiol 2007; 210: 757-65.

[18] Eidelman F, Fuks A, DeMarte L, Taheri M, Stanners C. Human carcinoembryonic antigen, an intercellular adhesion molecule, blocks fusion and differentiation of rat myoblasts. J Cell Biol 1993; 123: 467-75.

[19] Ordoñez C, Screaton R, Ilantzis C, Stanners C. Human carcinoembryonic antigen functions as a general inhibitor of anoikis. Cancer Res 2000; 60: 3419-24.

[20] Ilantzis C, Jothy S, Alpert L, Draber P, Stanners C. Cell-surface levels of human carcinoembryonic antigen are inversely correlated with colonocyte differentiation in colon carcinogenesis. Lab Invest 1997; 76: 703-16.

[21] Bowcock A, Krueger J. Getting under the skin: the immunogenetics of psoriasis. Nat Rev Immunol 2005; 5: 699-711.

[22] Han S, Kwak T, Her K, et al. CEACAM5 and CEACAM6 are major target genes for Smad3-mediated TGF- $\beta$ signaling. Oncogene 2008; 27: 675-83. 